\title{
DECORRELATION EFFECTS IN BISTATIC TANDEM-X DATA
}

\author{
Michele Martone, Benjamin Bräutigam, Gerhard Krieger \\ German Aerospace Center (DLR), Microwaves and Radar Institute, Oberpfaffenhofen, Germany
}

\begin{abstract}
The TanDEM-X mission comprises two nearly identical satellites: TerraSAR-X and TanDEM-X. The primary objective of the mission is the generation of a worldwide and consistent digital elevation model (DEM) with an unprecedented accuracy. The two satellites fly in a close orbit configuration and act as a large single-pass radar interferometer with the opportunity for flexible baseline selection, allowing the acquisition of highly accurate cross- and along-track interferograms [1]. One of the key parameters in estimating interferometric performance is the coherence $\gamma$. Several error sources may contribute to a coherence loss: in this paper, the impact of limited signal-to-noise ratio (SNR), volume decorrelation, and quantization errors are investigated in detail. Furthermore, a novel azimuthswitched quantization (ASQ) technique is introduced and a preliminary performance assessment is presented.
\end{abstract}

Index Terms - Bistatic synthetic aperture radar (SAR), interferometry, coherence, digital elevation model (DEM), TanDEM-X, azimuth-switched quantization (ASQ).

\section{INTRODUCTION}

In the last decades, an increasing interest has arisen to use Interferometric Synthetic Aperture Radar (InSAR) systems in remote sensing applications. InSAR exploits the phase difference of (at least) two complex SAR images, acquired from different orbit positions and/or at different times. This derived information allows the estimation and assessment of many geophysical parameters, such as ocean currents, ground deformations, and Earth's topography by generation of digital elevation models (DEMs). InSAR is nowadays a wellrecognized and powerful instrument and DEMs are widely employed in many commercial and scientific applications, such as, for example, Global Positioning System (GPS), as well as many geoscience fields, like geology, physical geography, glaciology, and oceanography. TanDEM-X (TerraSAR-X add-on for Digital Elevation Measurement) is the first operational spaceborne bistatic SAR system and is based on two twin satellites: TerraSAR-X (TSX, launched in 2007), and TanDEM-X (TDX, launched in June 2010). In December 2010, TanDEM-X began with operational global acquisition: bistatic and monostatic SAR images are simultaneously acquired in stripmap mode and processed to interferograms. The primary objective of the mission is the generation of a global, consistent and highprecision DEM [1]. This goal will be achieved by performing at least two global mappings of the Earth's landmass. The key quantity in estimating interferometric performance is the coherence $\gamma$, which represents the normalized correlation coefficient between the master and slave acquisitions and gives information about the amount

The TanDEM-X project is partly funded by the German Federal Ministry for Economics and Technology (Förderkennzeichen 50 EE 1035).

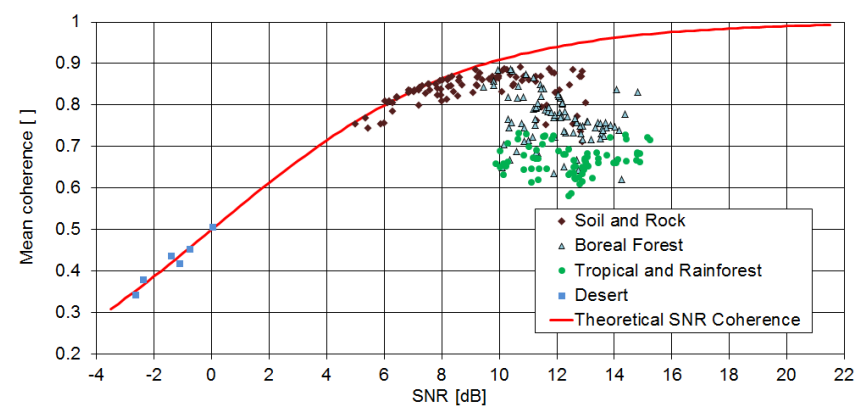

Fig. 1. Interferometric coherence over Signal-to-Noise Ratio (SNR). The red line indicates the theoretical SNR coherence.

of noise in the interferogram [2]. Several error sources may contribute to coherence loss. This paper presents a detailed evaluation of decorrelation effects in bistatic TanDEM-X data and is organized as follows. First, the impact of limited signal-to-noise ratio (SNR) is assessed. Volume decorrelation effects are evaluated in Section 3 , by means of statistical analyses and dedicated acquisitions on defined test sites, and the strategies to handle with those areas strongly affected by decorrelation phenomena are presented. In Section 4 the quantization effects on coherence are investigated. In the context of the scalar quantization algorithms, a novel azimuth-switched quantization (ASQ) technique is also illustrated, which allows the implementation of non-integer quantization rates without any increase of the complexity and computational load.

\section{SNR DECORRELATION}

The signal-to-noise ratio (SNR) is a measure to describe the image quality of remote sensing systems, estimating how much a signal has been corrupted by noise. From a SAR image, the SNR can be computed as follows

$$
\mathrm{SNR}=\frac{\beta_{0} \cdot \sin \left(\theta_{i}\right)-\mathrm{NESZ}}{\mathrm{NESZ}},
$$

where $\beta_{0}$ is the calibrated radar brightness, $\theta_{i}$ is the incidence angle, and NESZ stands for Noise Equivalent Sigma Zero, which describes the influence of noise contributions, like antenna pattern, instrument thermal noise, and processing filters. On TSX and TDX, it is estimated by means of receive-only pulses, which are acquired during the data-take. In order to investigate the effects of backscatter intensity on interferometric performance, acquisitions over areas showing different vegetation and soil characteristics have been analyzed. In Figure 1 the interferometric coherence over SNR is depicted. Most of the land cover types show in general good performance in terms of SNR (higher than $6 \mathrm{~dB}$ ) and coherence rarely below 0.6 , which 


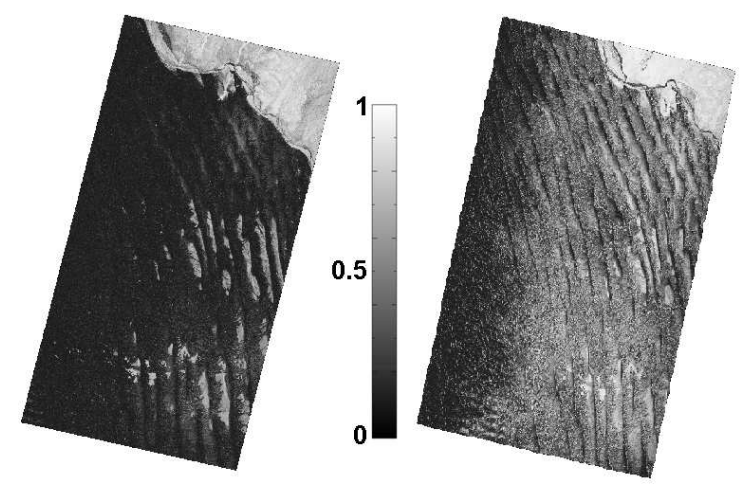

Fig. 2. Coherence maps over Namibia desert; (left) $\mathrm{HH}$ polarization, HoA: $45 \mathrm{~m}\left(\mathrm{~B}_{\perp}: 186 \mathrm{~m}\right), \theta_{i}: 47.7^{\circ}$, mean coherence (over sand) $\sim 0.25$; (right) $\mathrm{HH}$ polarization, $\mathrm{HoA}: 26 \mathrm{~m}\left(\mathrm{~B}_{\perp}: 162 \mathrm{~m}\right), \theta_{i}$ : $32.4^{\circ}$, mean coherence (over sand) $\sim 0.41$. On the rocky area, the mean coherence is $\sim 0.7$ for both images.

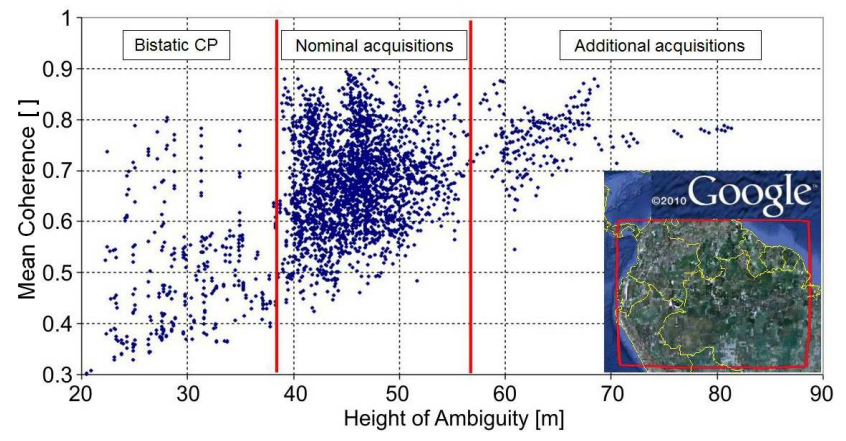

Fig. 3. Mean coherence over height of ambiguity (HoA) for about 3000 bistatic scenes acquired over Amazon rainforest: test acquisitions with $\mathrm{HoA}<38 \mathrm{~m}$ (during the bistatic Commissioning Phase, left part of the image); nominal acquisitions with target $\mathrm{HoA}=45$ $\mathrm{m}$ (central part), $\bar{\gamma}=0.68$; re-acquisition of affected data takes with HoA > 57 m (right part), $\bar{\gamma}=0.76$.

provides in most cases sufficient phase unwrapping quality. The red line on the top of the figure indicates the theoretical SNR coherence, i.e. the expected coherence as if the finite sensitivity of the receiving antenna was the only error source [2], [3]. On the other hand, the performance is strongly deteriorated over regions covered by sandy deserts, mainly due to the weak power of the backscattered signal: for SNR lower than $2 \mathrm{~dB}$, coherence usually smaller than 0.5 is observed. In Figure 2, as an example, the coherence maps for two bistatic acquisitions over the Namibia desert are shown. For the image on the left side, the incidence angle is $48^{\circ}$, for the one on the right side it is $32^{\circ}$. Looking at the single SAR images the SNR is about $3 \mathrm{~dB}$ higher in the right image, and a coherence improvement of about $60 \%$ (from 0.25 to 0.41 ) is obtained over the sandy area. Strong dependency of interferometric performance on the incidence angle has been systematically observed for all the investigated test sites over desert. Therefore, the acquisition geometry of the second global acquisition has been consequently optimized for such low backscatter areas, by employing, where possible, steeper incidence angles in order to minimize the performance loss [4]. The influence

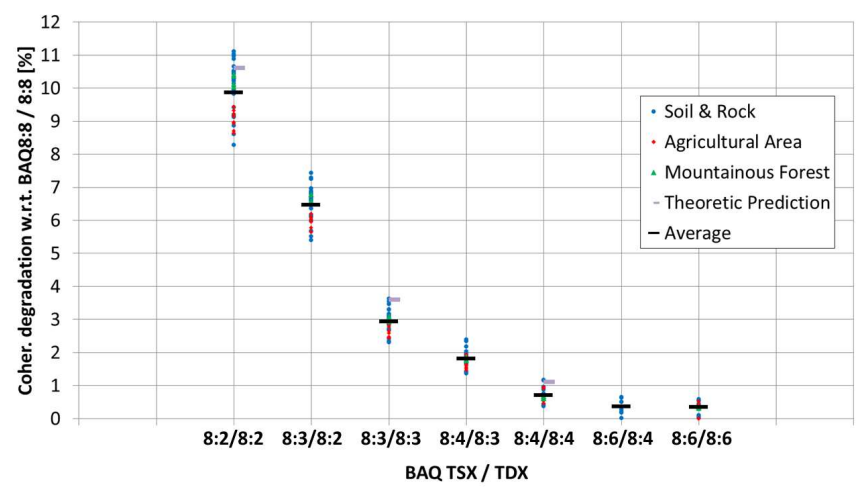

Fig. 4. Coherence degradation (in \%) with respect to BAQ 8:8/8:8, for different land cover types.

of other aspects which might affect the NESZ estimation as, e.g., data quantization, is presently under study and will be further investigated.

\section{VOLUME DECORRELATION}

On vegetated areas, volume scattering is a major source of coherence loss. The existence of multiple scatterers at different heights and within a single resolution cell results in an increase of the interferometric phase uncertainty. In Figure 3, coherence over height of ambiguity (HoA) from around 3000 bistatic acquisitions over the Amazon rainforest is shown (the considered area is highlighted in the lower right corner). The height of ambiguity is defined as the height difference corresponding to a complete $2 \pi$ cycle of the interferometric phase. It is in inverse proportion to the effective baseline and tells about the phase-to-height sensitivity in the interferogram. Test acquisitions with HoA smaller than $38 \mathrm{~m}$ (left part of the image) were commanded during the bistatic Commissioning Phase (between October and December 2010), and a critical performance can be observed, with $\gamma$ rarely above 0.6 . For this reason, a new target height of ambiguity for the first global DEM acquisition was set to $45 \mathrm{~m}$ (initially set at $40 \mathrm{~m}$ ). The corresponding points in Figure 3 lie between the two vertical lines. Here, a mean coherence of about 0.68 is observed, which is already expected to provide sufficient phase unwrapping quality. Adapting the TanDEM-X acquisition plan, the order of helix satellite formations was reversed, starting with larger baselines, so that affected data could be successively re-acquired with a smaller baseline (i.e. larger HoA) [5]. However, one has to be aware that the height of ambiguity is a direct scaling factor of the relative height error, and therefore it should be kept as small as possible. Such additional acquisitions are depicted on the right part of Figure 3, and a clearly visible coherence improvement is observed. The combination of additional and nominal acquisitions, by means of multi-baseline phase unwrapping [6], will then allow to fully meet the final DEM specifications.

\section{QUANTIZATION DECORRELATION}

In SAR applications, raw data quantization represents an essential aspect, since the number of bits employed for radar signal digitization affects on the one hand the memory consumption of the satellite and the data volume to be transmitted to the ground, but also on 


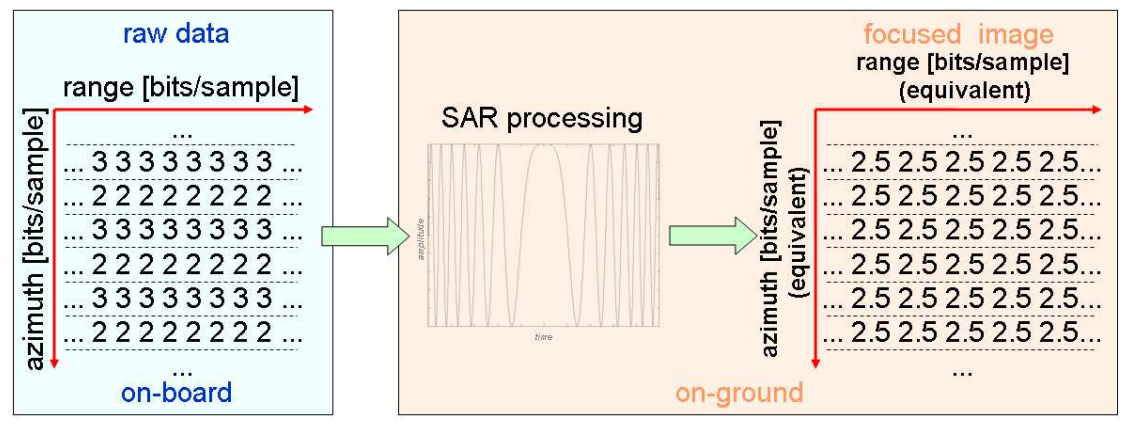

Fig. 5. Workflow for ASQ implementation on a SAR system. Integer rates are switched along the azimuth direction during the data take. After SAR processing, the focused image will show a performance equivalent to as if the raw data was acquired with an "average" of the original sequence. In this example, a fractional rate of 2.5 bits/sample is obtained as a result of switching between 2 and 3 bits/sample, range line by range line.

the other hand the performance of the SAR images. On TerraSAR$\mathrm{X}$ and TanDEM-X the received signal is first digitized by an 8-bit analog-to-digital converter (ADC), and then a block adaptive quantizer (BAQ) is employed for raw data compression. Possible compression rates are 8:2 (from 8 to 2 bits/sample), 8:3, 8:4, 8:6 and 8:8, where the latter corresponds to BAQ bypass. In order to evaluate the impact of quantization on the coherence, raw data commanded with BAQ 8:8 have been synthetically recompressed on ground by using available BAQ rates. The obtained products have then been processed into SAR images by the Interferometric TanDEM-X Processor [7], allowing the assessment of interferometric performance. In Figure 4 the quantization decorrelation with respect to the bypass case is plotted, for test sites showing different land cover characteristics acquired with varying polarization, incidence angles and baselines. As expected, the fewer bits are employed for quantization, the more coherence degradation is observed for the same test site. A coherence loss of about $1 \%$ and $3.5 \%$ is noticed by using 4 and 3 bits/sample, respectively, and good agreement between theoretic prediction [1] and mean experimental results is observed (marked with violet and black lines, respectively). A dedicated analysis aimed at the assessment of the quantization effects in presence of inhomogeneities in the backscatter distribution (the so-called low scatterer suppression [8]) is currently being performed. On those regions showing very good interferometric performance (i.e. $\gamma>0.8$ ), the opportunity to further increase the raw data compression rate in order to improve the overall DEM performance has been also exploited. In particular, BAQ 8:3 on one satellite and BAQ 8:2 on the other have been employed, resulting in a mean coherence degradation between $6 \%$ and $7 \%$ (with reference to the bypass case), against a considerable data rate reduction of about $17 \%$.

\subsection{Azimuth-Switched Quantization (ASQ) for SAR Systems}

In the context of scalar quantization techniques, compression of SAR data is calculated and commanded for whole received channels, as polarization channels, or for certain time spans of data acquisition. In the last years, non-integer quantization rates have been implemented, by following the quantizer with additional software/hardware blocks, at a cost of increasing the overall system complexity [9], [10]. An azimuth-switched quantization (ASQ) concept is described in the following: during the data collection by the SAR system, integer compression rates are toggled along the azimuth direction, at every echo range pulse (within a range line the quantization rate is kept constant). After performing the azimuth focusing, an equivalent non-integer quantization rate is synthesized, which results from an "average" of the different rates used. Highly flexible adaptation of SAR performance and calculation of memory resources are then possible, and only a simple quantizer with integer rates is required (the capability of changing acquisition parameters during a SAR data take is a feature normally made available by the present state of technology). In Figure 5 the workflow is depicted for the case of 2.5 bits/sample, obtained by switching between 2 and 3 bits/sample at every range line. The compression rate can be theoretically changed at every pulse repetition interval (PRI), but the range lines to be taken into account for determining the effective average quantization rate are those "lying" inside one synthetic aperture: for typical spaceborne SAR scenarios, with the presented technique bit rates until a fraction of a thousandth of a bit can be effectively implemented. In Figure 6 the interferometric coherence is depicted for rates between 2 and 3 bits/sample (the corresponding compression ratios are also given on the upper part of the figure) and for different test sites. Each curve describes the performance of one acquisition, and an almost linear coherence trend can be observed. In particular, it could be verified that the performance obtained in the case of 2.5 bits/sample is in practice the same as that using 3 bits/sample on one satellite and 2 bits/sample on the other. In order to keep the coherence loss unbiased along azimuth, the distribution of switched rates into an azimuth sequence has to be as uniform as possible. The sequences used for the presented analysis are listed in Table 1.

Looking at single SAR images, one could benefit from the implementation of fractional bit rates in order to reduce the impact, among the others, of the azimuth ambiguities. In order to satisfy the sampling requirement, the pulse repetition frequency (PRF) must be sufficiently high. In general, the higher is the PRF, the smaller are the azimuth ambiguities. Therefore, given a fixed amount of data to be stored (which often represents a bottleneck for spaceborne SAR systems), a reduced ambiguous signal power can be obtained by reducing the quantization rate (i.e., by increasing the PRF).

\section{CONCLUSIONS}

This paper presented a coherence evaluation of TanDEM-X interferometric data. It was shown that sites characterized by differ- 


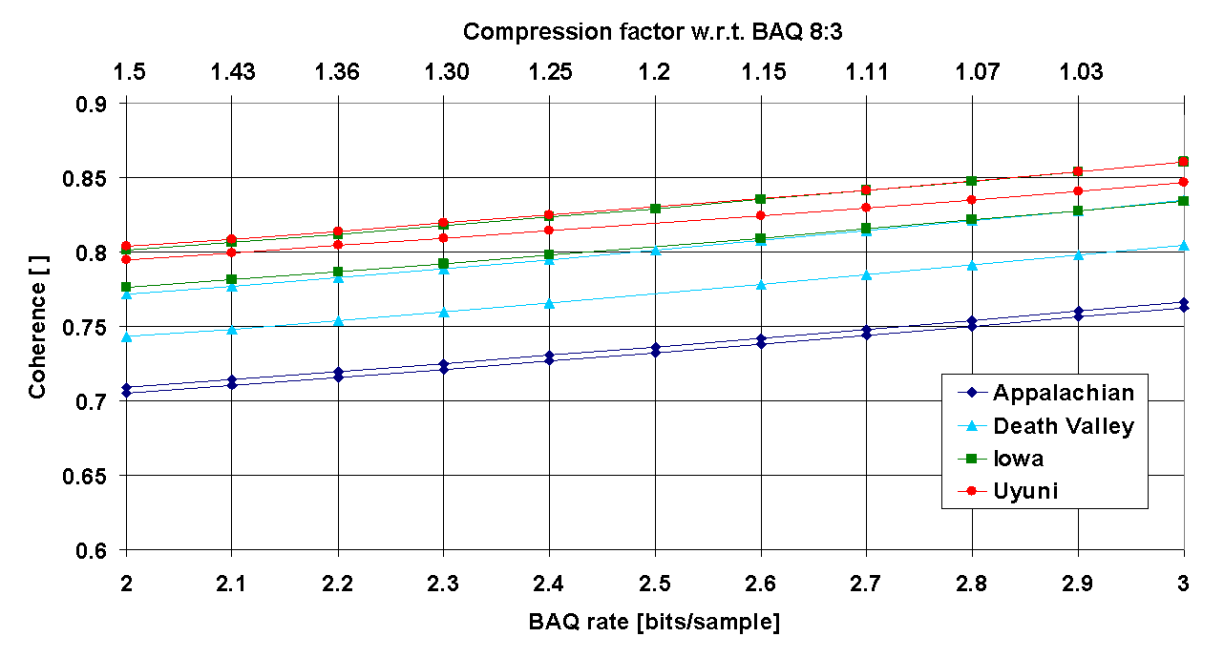

Fig. 6. Interferometric coherence over non-integer compression rates between 2 and 3 bits/sample, for different test sites (depicted with different colors). Each curve corresponds to one acquisition. On a conventional system only the rates 2 and 3 bits/sample are available.

Table 1. Example of fractional quantization rates and relative azimuth sequences.

\begin{tabular}{|c|c|}
\hline Compression Rate [bits/sample] & ASQ Sequence \\
\hline 2.1 & 222222222223 \\
\hline 2.2 & 2222233222223 \\
\hline 2.3 & 223322322232 \\
\hline 2.4 & 23223323223 \\
\hline 2.5 & 23232232323 \\
\hline 2.6 & 23233232333 \\
\hline 2.7 & 2323332333 \\
\hline 2.8 & 23333323333 \\
\hline 2.9 & 2333333333 \\
\hline
\end{tabular}

ent backscatter intensities show satisfactory and reproducible interferometric performance, and a strategy to handle with those areas strongly affected by SNR decorrelation has been presented. The influence of other aspects which might affect the NESZ estimation as, e.g., data quantization, is currently being investigated. Due to volume decorrelation effects, dense forests require additional acquisitions with an optimized imaging geometry (i.e. smaller baselines) and, thus, the DEM acquisition plan has been consequently adapted, by increasing the target HoA to $45 \mathrm{~m}$ and planning additional acquisitions with a height of ambiguity over $60 \mathrm{~m}$ in critical areas. Quantization causes acceptable performance loss and good agreement has been observed between experimental results and theoretical prediction [1]. The opportunity to further increase the raw data compression rate (e.g., different rates between the two satellites) in order to improve the overall DEM performance has been exploited. An evaluation of the quantization effects in presence of inhomogeneities in the backscatter distribution is ongoing. A novel azimuth-switched quantization (ASQ) technique has been presented and a preliminary performance assessment has been conducted.

\section{REFERENCES}

[1] G. Krieger, A. Moreira, H. Fiedler, I. Hajnsek, M. Werner, M. Younis, and M. Zink: TanDEM-X: A Satellite Formation for
High-Resolution SAR Interferometry, IEEE Trans. Geosci. Remote Sens., vol. 45, no. 11, pp. 3317-3341, Nov. 2007.

[2] H. A. Zebker, and J. Villasenor: Decorrelation in Interferometric Radar Echoes, IEEE Trans. Geosci. Remote Sens., vol. 30, no. 5, pp. 950-959, Sep. 1992.

[3] D. Just, and R. Bamler: Phase Statistics of Interferograms with Applications to Synthetic Aperture Radar, Appl. Opt., vol 33, no. 20, pp. 4361-4368, Jul. 1994.

[4] C. Ortega-Mìguez, D. Schulze, M. D. Polimeni, J. Böer, P. Rizzoli, and M. Bachmann: TanDEM-X Acquisition Planner, in EUSAR, Nuremberg, Germany, 2012.

[5] B. Bräutigam, M. Martone, P. Rizzoli, M. Bachmann, and G. Krieger: Interferometric Performance of TanDEM-X Global DEM Acquisitions, in EUSAR, Nuremberg, Germany, 2012.

[6] M. Lachaise, M. Eineder, and T. Fritz: Multi Baseline SAR Acquisition Concepts and Phase Unwrapping Algorithms for the TanDEM-X Mission, in Proc. IGARSS, Barcelona, Spain, 2007, pp. $5272-5276$.

[7] T. Fritz, C. Rossi, N. Yague-Martinez, F. Rodriguez-Gonzalez, M. Lachaise, and H. Breit: Interferometric Processing of TanDEM-X Data, in Proc. IGARSS, Vancouver, Canada, 2011, pp. $2428-2431$.

[8] S. Huber, M. Younis, and G. Krieger: The TanDEM-X Mission: Overview and Interferometric Performance, Int. J. Microw. Wireless Technol., pp. 1-11, 2010.

[9] T. Algra: Data Compression for Operational SAR Missions Using Entropy-Constrained Block Adaptive Quantization, in Proc. IGARSS, Toronto, Canada, 2002, pp. 1135-1139.

[10] P. Guccione, and A. M. Guarnieri: A Space Adaptive Quantizer for Spaceborne SAR, IEEE Trans. Geosci. Remote Sens., vol. 49, no. 10, pp. 3564-3573, Oct. 2011. 